\title{
A Compressive Superresolution Display
}

\author{
Felix Heide ${ }^{1}$, James Gregson ${ }^{1}$, Gordon Wetzstein ${ }^{3}$, Ramesh $_{\text {Raskar }}{ }^{3}$ and \\ Wolfgang Heidrich ${ }^{1,2}$ \\ 1: University of British Columbia, 2: King Abdullah University of Science and Technology and 3: MIT Media Lab
}

\begin{abstract}
In this paper, we introduce a new compressive display architecture for superresolution image presentation that exploits co-design of the optical device configuration and compressive computation. Our display allows for superresolution, HDR, or glasses-free 3D presentation.
\end{abstract}

OCIS codes: $120.2040,080.1753$.

\section{Introduction}

Throughout the last few years, display technology has undergone a major transformation. Whereas improvements of display characteristics, such as resolution and contrast, have traditionally relied on advances in optical and electrical fabrication, computation has become an integral part of the image formation. Through the co-design of display optics and computational processing, computational and compressive displays have the potential to overcome fundamental limitations of purely optical designs.

In this paper, we explore a novel compressive superresolution display design employing only off-the-shelf hardware components: two high-speed liquid crystal displays (LCDs) are mounted, with a slight offset, on top of each other. While this setup is inspired by previously proposed light field displays [5], we introduce an additional diffuser covering the LCD closest to the observer, which combines the respective contribution of the panels into a single superresolved, two-dimensional image (see Fig. 1).

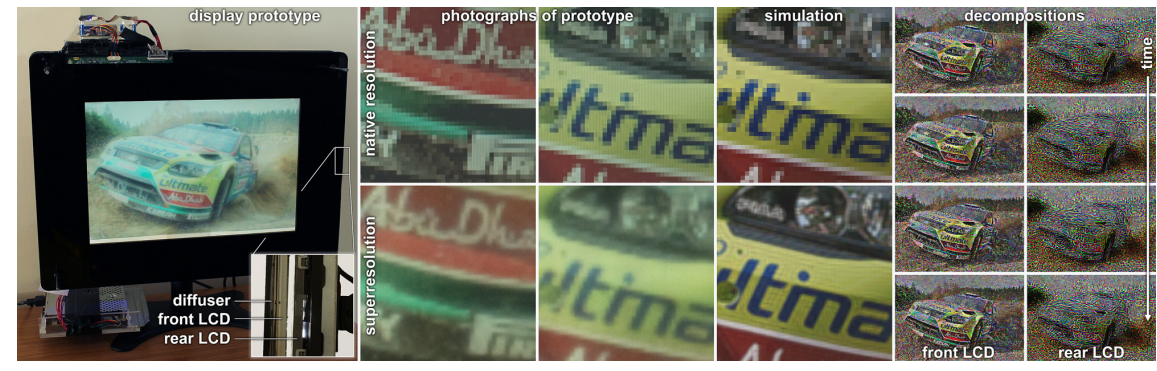

Fig. 1. Compressive superresolution display. The proposed display architecture comprises two stacked high-speed liquid crystal displays (LCDs) covered by a diffuser (left). A target highresolution image is then decomposed into a set of patterns that are shown on the front and rear LCD in quick succession (right). Compared to the native resolution of each panel (center, top row), the proposed compressive display approach achieves significant improvements in resolution (center, bottom row) without any mechanically moving parts.

The key insight of our work is that the two stacked LCDs synthesize an intermediate light field inside the device; the diffuser then integrates the different views of that light field such that an observer perceives a superresolved, twodimensional image. Previously-proposed superresolution displays also exploit integration of different images either optically by superimposing multiple projections on the same screen (e.g., [3]) or perceptually by displaying different patterns on a quickly moving device (e.g., [1]). By removing the need for multiple projection devices and mechanically moving parts, the display proposed in this paper is well-suited for flat-screen televisions and mobile devices.

The diffuser in our system can be electronically switchable, which allows not only for superresolution image display, but also for a glasses-free 3D as well as a high dynamic range display mode (see Fig. 2).

We make the following contributions:

- We introduce a display design that combines a nonlinear convex optimization framework with a duallayer device configuration for the application of superresolved image generation. 
Compressive Superresolution Display

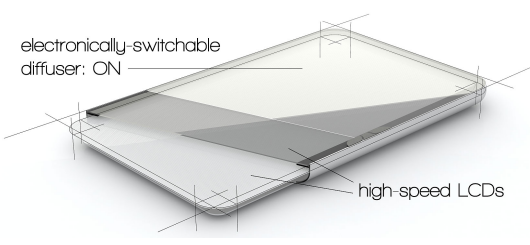

Compressive Light Field Display

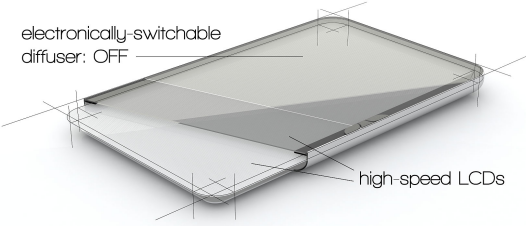

High Dynamic Range Display

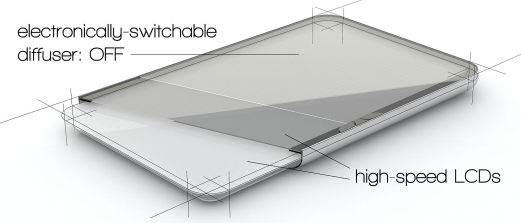

Fig. 2. Conceptual sketch of a multi-purpose compressive display. The device consists of two highspeed liquid crystal panels covered with an electronically-switchable diffuser. With the diffuser being activated, the display is capable of showing superresolved 2D images (left). The diffuser can be deactivated and becomes transparent. In this configuration, the device can emit either a light field for glasses-free 3D display (center) or a high dynamic range image (right).

- We analyze display design parameters and evaluate the proposed system in simulation and with a prototype device.

- We demonstrate that the proposed hardware design is flexible enough to support a variety of display modes, including a previously documented light field mode and a new, low-rank, high dynamic range mode.

\section{Image formation and optimization}

In this section, we derive models for superresolved image formation, via compressive light field display, and inverse methods to compute optimal pixel states for a target high-resolution image.

\subsection{Optical Image Formation}

The proposed optical display configuration comprises a light field display behind a diffuser. The image $i(\mathbf{x})$ observed on the diffuser is a projection of the incident light field $l(\mathbf{x}, v)$ over the angular domain $\Omega_{v}$ :

$$
i(\mathbf{x})=\int_{\Omega_{v}} l(\mathbf{x}, v) d v .
$$

Here, $\mathbf{x}$ is the $2 \mathrm{D}$ spatial coordinate on the diffuser and $v$ denotes the angle. The light field absorbs angle-dependent integration weights of the diffuser. The light field that is synthesized inside the display and incident on the diffuser is

$$
\tilde{l}(\mathbf{x}, v)=\frac{1}{K} \sum_{k=1}^{K} f^{(k)}(\mathbf{x}-d \cdot v) \cdot g^{(k)}\left(\mathbf{x}-\left(d+d_{l}\right) \cdot v\right),
$$

where $d$ is the distance between diffuser and front panel and $d_{l}$ is the distance between front and rear panel (Fig. 3, left). The spatial coordinates on the panels are denoted by $\xi$ whereas the functions $f\left(\xi_{1}\right)$ and $g\left(\xi_{2}\right)$ give the transmittance of front and rear panel at each position.

In this model, the panels run at a frame rate that is $K$ times faster than the HVS. As observed by Wetzstein et al. [5], the emitted light field of a pair of LCD patterns corresponds to their outer product and is therefore rank-1. The light field observed from the high-speed panels $\tilde{l}(\mathbf{x}, v)$ is rank- $K$ due to the retinal integration of $K$ rank-1 light fields. Combining Equations 1 and 2 results in the following expression for the image observed on the diffuser:

$$
\tilde{i}(\mathbf{x})=\int_{\Omega_{v}} \frac{1}{K} \sum_{k=1}^{K}\left(f^{(k)}(\mathbf{x}-d \cdot v) \cdot g^{(k)}\left(\mathbf{x}-\left(d+d_{l}\right) \cdot v\right)\right) d v=\frac{1}{K} \sum_{k=1}^{K} \iint \phi\left(\mathbf{x}-\xi_{1}, \mathbf{x}-\xi_{2}\right)\left(f^{(k)}\left(\xi_{1}\right) \cdot g^{(k)}\left(\xi_{2}\right)\right) d \xi_{1,2} .
$$

Equation 3 shows that each location on the diffuser integrates over some area on front and rear LCD. This integration is modeled as a convolution with a $4 \mathrm{D}$ kernel $\phi$. Discretizing Equation 3 results in

$$
\mathbf{i}=\mathbf{P} \operatorname{vec}\left(\mathbf{F G}^{T}\right) .
$$

Here, the $K$ time-varying patterns of front and rear LCD panels are encoded in the columns of matrices $\mathbf{F} \in \mathbb{R}^{M \times K}$ and $\mathbf{G} \in \mathbb{R}^{M \times K}$, respectively (see Fig. 3, right). The resolution of the observed image $\mathbf{i} \in \mathbb{R}^{N}$ is larger than that of either panel, i.e. $N \geq M$. The convolution kernel is encoded in a discrete projection matrix $\mathbf{P} \in \mathbb{R}^{N \times M^{2}}$ and $\operatorname{vec}(\cdot)$ is a linear operator that reshapes a matrix into a vector by stacking up its rows. 

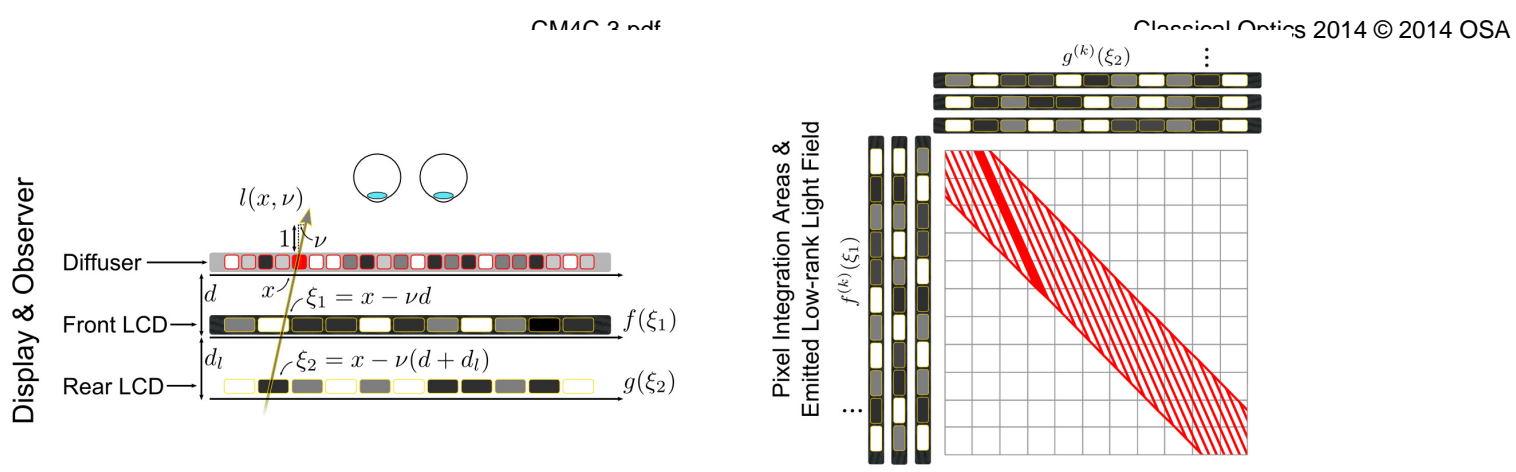

Fig. 3. Schematic of display components and parameters (left). A diffuser is directly observed by the viewer and optically projects a 4D light field into a superresolved 2D image. The light field is emitted by two high-speed LCD panels. Optically, their combined effect is a multiplication allowing the light field to be represented by the outer product of their respective patterns $f\left(\xi_{1}\right)$ and $g\left(\xi_{2}\right)$ (right). The pixels on the diffuser have a resolution exceeding that of either LCD panel, their integration areas are illustrated in red (bottom).

\subsection{Superresolution Image Synthesis}

Given a target high-resolution image $\mathbf{i}$ and the image formation derived in the last subsection, we can formulate an objective function that minimizes the $\ell_{2}$-norm between target and emitted image given physical constraints of the pixel states

$$
\begin{array}{cl}
\underset{\{\mathbf{F}, \mathbf{G}\}}{\operatorname{minime}} & \left\|\mathbf{i}-\mathbf{P} \operatorname{vec}\left(\mathbf{F G}^{T}\right)\right\|_{2}^{2} \\
\text { s.t. } & 0 \leq \mathbf{F}, \mathbf{G} \leq 1
\end{array}
$$

This objective is difficult to deal with, as it involves a large matrix factorization embedded within a deconvolution problem. We therefore introduce a splitting variable for the intermediate lightfield $\mathbf{F G}^{T}$ and then solve Equation 5 using the alternating direction method of multipliers (ADMM [2]). Please see our supplemental material for a derivation [4].

\section{Results}

Figure 1 shows simulated results as well as results using our prototype. For more protoype results and also detailed analysis using simulation please see our supplemental material [4].

\section{Conclusion}

In summary, we present a computational display approach to superresolution image synthesis. Through the co-design of display optics and computational processing, the proposed architecture is the first to facilitate superresolution with a single device and without the need for mechanically moving parts in a form factor suitable for televisions and computer monitors. We analyze the system simulation and with a prototype. Further, we demonstrate that the display can be operated in different modes to facilitate either superresolution image display, high dynamic range display, or light field synthesis by simply changing the driver software.

\section{References}

1. Floraine Berthouzoz and Raanan Fattal. Resolution Enhancement by Vibrating Displays. ACM Trans. Graph., 31(2):15:1-15:14, 2012.

2. Stephen Boyd, Neal Parikh, Eric Chu, Borja Peleato, and Jonathan Eckstein. Distributed optimization and statistical learning via the alternating direction method of multipliers. Foundations and Trends in Machine Learning, 3(1):1-122, 2011.

3. Niranjan Damera-Venkata and Nelson L. Chang. Display Supersampling. ACM Trans. Graph., 28(1):9:1-9:19, 2009.

4. Felix Heide, James Gregson, Gordon Wetzstein, Ramesh Raskar, and Wolfgang Heidrich. Supplement of this paper: http://cs.ubc.ca/ fheide/SuperResolution.pdf. 2014.

5. Gordon Wetzstein, Douglas Lanman, Matthew Hirsch, and Ramesh Raskar. Tensor Displays: Compressive Light Field Synthesis using Multilayer Displays with Directional Backlighting. ACM Trans. Graph. (SIGGRAPH), 31(4):80, 2012. 